\title{
Thaparotrema akbari new species of genus Thaparotrema Gupta, 1955 from host Rita rita (Siluriformes: Bagridae) of River Indus Sindh Pakistan
}

Hira Soofi ${ }^{1 *}$, Nadir Ali Birmani ${ }^{1}$, Ali Murtaza Dharejo ${ }^{1}$, Abdul Rasool Abbasi $^{2}$ and Ghulam Sarwar Ghachal ${ }^{1}$

1. Department of Zoology, University of Sindh, Jamshoro, Sindh-Pakistan

2. Department of Fresh Water Biology and Fisheries, University of Sindh, Jamshoro, Sindh-Pakistan

*Corresponding author's email: hirasoofi@gmail.com

Citation

Hira Soofi, Nadir Ali Birmani, Ali Murtaza Dharejo, Abdul Rasool Abbasi and Ghulam Sarwar Ghachal. Thaparotrema akbari new species of genus Thaparotrema Gupta, 1955 from host Rita rita (Siluriformes: Bagridae) of river Indus Sindh Pakistan. Pure and Applied Biology. Vol. 10, Issue 1, pp254-261. http://dx.doi.org/10.19045/bspab.2021.100026

Received: 22/06/2020 Revised: 24/08/2020

Accepted: 26/08/2020

Online First: 17/09/2020

\section{Abstract}

During current study on order Siluriformes belong to diverse group of ray-finned fishes. Rita rita Siluriformes catfish collected from River Indus Jamshoro, Pakistan were bought to Parasitology laboratory of zoological department. Fishes were dissected for examined of helminth parasites. Total 12 specimens of genus Thaparotrema were collected from gallbladder of hosts and processing through standard method. Diagram were made and identify with the help of keys and literature. The new species Thaparotrema akbari identified on the basis following differential characters having large body size, elongate and narrow shape, oral sucker elongate and egg shape, ventral sucker wheel shaped, pharynx oval shape, anterior testis rounded to quadrangular in shape, posterior testis pentamerous in shape with broad anterior and narrow posterior end, ovary heart shape or triangular, seminal receptacle quadrangular tube shape and vertical in position, uterine coils almost overlapped caecum and whole middle region of body, vitelline are in small follicle.

Keywords: Catfish; Indus Rive; Rita rita; Siluriformes; Trematodes; Thaparotrema akbari

\section{Introduction}

Helminths of genus Thaparotrema are parasites of gallbladder and intestine of freshwater fishes, they cause harmful impact on fish health and nutritional value. Type species of Thaparotrema genus is Thaparotrema vittalani collected from intestine and gallbladder of fish Rita rita from India $[1,2]$. Other species of genus
Thaparotrema recorded from world are Thaparotrema pedicellatum [1-4] collected from intestine and gallbladder of fishes Rita rita, Hemibagrus nemurus and Rita buchanani from Thailand and Pakistan; Thaparotrema piscicola collected from gallbladder of Gymnarchus niloticus from Africa [1, 2, 4]; Thaparotrema botswanensis collected from gallbladder of Clarias 
gariepinus from Africa $[5,6]$. Throughout the world the helminths are reported from variety of animals as well as from fishes [7]. Various form of infection cause in fishes depending upon habitat of helminth parasites [8]. Reports on helminths of Siluriformes order catfishes in Pakistan are limited [3, 8-14]. Present work on helminths of Siluriformes order catfishes in Pakistan has great importance in field of taxonomy and fishery.

\section{Materials and Methods}

Rita rita host fishes were collected from River Indus Jamshoro, Pakistan and bought to the Parasitology laboratory. Host were dissected and visceral organs examined for helminth parasites under dissecting microscope. Total 12 specimens were collected hosts, for collection and processing of trematodes standard method were followed [15, 16]. Trematodes were fixed and kept in $70 \%$ ethanol for overnight, stained with borax carmine, dehydrated in graded series of ethanol, cleared in clove oil and xylol and mounted in Canada balsam. Diagram were made by Camera Lucida and photographs were taken with the help of Olympus DP12 Camera, identification with the help of keys and literature. All measurement were taken in millimeters.

\section{Result}

Thaparotrema akbari n.sp.

Family: Opisthorchiidae Braun 1901

Genus: Thaparotrema Gupta 1955

Type host: Rita rita

Site of infection: Gallbladder

Type locality: River Indus at Jamshoro, Sindh, Pakistan

Number of specimen: 12

Specimens: Deposit in Department of Zoology, University of Sindh, Jamshoro.

Etymology: The name of new species refers to the honored of author's father name Soofi Akbar.

\section{Description}

The description of specimen base on figure 1 and 2. Body of parasite elongate, narrow, curved at many regions, spindle shape measures 5.6-5.36 X 0.5-0.6, anterior region of body long and narrow, middle region long and wider, again posterior region long and narrow. Widest at post-equatorial region of body. Tegument without spines. Oral sucker small, elongate, egg shape, terminal measures 0.02-0.07 X 0.16-0.17. Ventral sucker well developed, muscular, rounded wheel shaped and pre-equatorial measures 0.26-0.29 X 0.22-0.24. Prepharynx long measures $0.18-0.18 \quad X \quad 0.06-0.06$ and pharynx oval shape, vertical in position measures 0.04-0.05 X 0.1-0.1. Esophagus short, broad measures 0.04-0.06 X 0.08-0.09 and intestinal bifurcation near to anterior region just after the oral sucker. Caecum narrow, extended from pharynx to the ventral sucker and then overlapped by uterus. Testes two tandem, diagonal, postequatorial, anterior testis rounded to quadrangular in shape measures $0.28-0.35 \mathrm{X}$ $0.26-0.31$, posterior testis pentamerous in shape measures $0.36-0.36 \quad \mathrm{X} \quad 0.28-0.30$. Ovary heart shape or triangular, anterolateral to seminal receptacle, right side of body measures 0.16-0.19 X 0.22-0.22. Seminal receptacle pretesticular, quadrangular tube shape, vertical in position measures 0.12$0.13 \mathrm{X}$ 0.32-0.37. Uterine coils well developed, almost overlapped caecum and whole middle region, uterus from anterior end of body measures 1.26-1.27 and from posterior end of body measures 1.08-1.11. Vitelline follicle laterally fields from anterior region to ovary. Genital pore anterior to ventral sucker.

\section{Discussion}

The comparative measurement of present species with previously reported species of genus Thaparotrema given in table 1 and 2. Detail morphological difference given below: 
Thaparotrema vittalani $[1,2,4]$ collected from intestine of and gallbladder of fish Rita rita in India varies from new species by fusiform, pear shape body; widest at post equatorial region of body; tegument spined; oral sucker rounded; prepharynx absent; pharynx rounded; esophagus long; caeca extended from anterior region to posterior extremity of body, not overlapped by uterus; testes separated by excretory bladder, oval to rounded in shape; ovary rounded; laurel canal present; uterine coils not much compact, intra-caecal; vitelline follicles not much compact, extra-caecal, excretory bladder

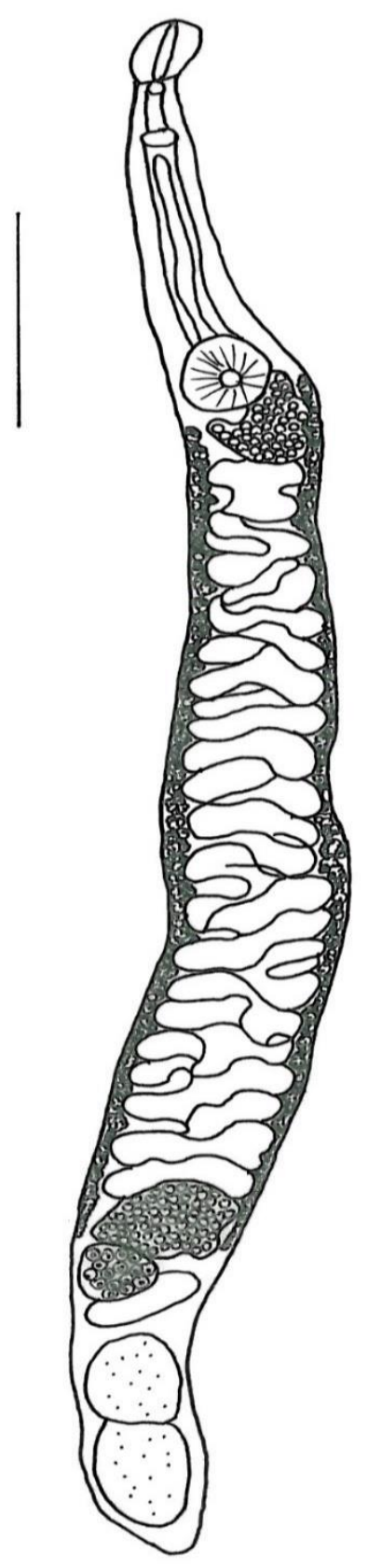

Figure 1. Thaparotrema akbari n.sp. Diagram of entire worm with scale bar of $1 \mathrm{~mm}$ 
Soofi et al.
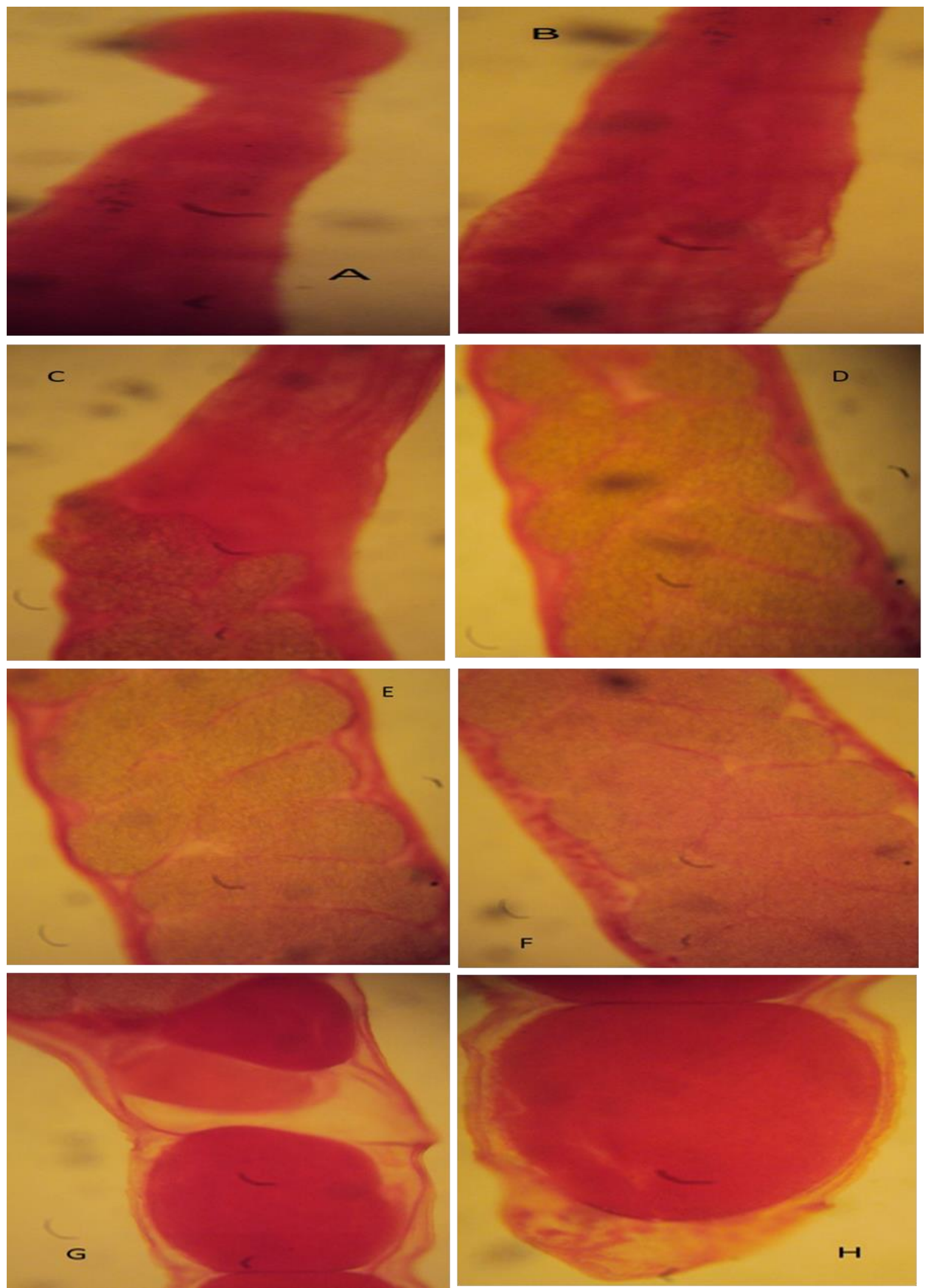

Figure 2. Thaparotrema akbari n.sp. A, B, C, D, E, F, G, H. Photographs of different parts of worm 
Table 1. List of comparative measurement of different species of genus Thaparotrema

\begin{tabular}{|c|c|c|c|c|}
\hline Species & $\begin{array}{c}\text { Thaparotrema } \\
\text { akbari n.sp. }\end{array}$ & $\begin{array}{c}\text { Thaparotrema } \\
\text { vittalani } \\
\text { Gupta } 1955\end{array}$ & $\begin{array}{c}\text { Thaparotrema } \\
\text { pedicellatum } \\
\text { Verma } 1927 \text { and } \\
\text { Soofi } \text { et al., } 2015 \\
\end{array}$ & $\begin{array}{c}\text { Thaparotrema } \\
\text { botswanensis } \\
\text { Rensburg et al., } \\
2013 \\
\end{array}$ \\
\hline Host & Rita rita & Rita rita & $\begin{array}{c}\text { Rita rita, } \\
\text { Hemibagrus } \\
\text { nemurus and Rita } \\
\text { buchanani }\end{array}$ & $\begin{array}{l}\text { Clarias } \\
\text { gariepinus }\end{array}$ \\
\hline Locality & Pakistan & India & $\begin{array}{l}\text { Pakistan, } \\
\text { Thailand }\end{array}$ & Africa \\
\hline Body & $5.6-5.36$ & 4.18 & $4.347-4.530$ & $1.74-4.80$ \\
\hline Width & $0.5-0.6$ & 0.58 & $0.759-0.80$ & $0.30-0.75$ \\
\hline Oral Sucker & $\begin{array}{c}0.02-0.07 \text { X } 0.16- \\
0.17\end{array}$ & $0.18 \times 0.15$ & $\begin{array}{c}0.230-0.280 \mathrm{X} \\
0.138-0.150\end{array}$ & $\begin{array}{c}0.17-0.44 \times 0.16- \\
0.45\end{array}$ \\
\hline Ventral Sucker & $\begin{array}{c}0.26-0.29 \times 0.22- \\
0.24\end{array}$ & $0.13 \times 0.15$ & $\begin{array}{c}0.115-0.135 \mathrm{X} \\
0.138-0.150\end{array}$ & $\begin{array}{c}0.14-0.35 \times 0.15- \\
0.34\end{array}$ \\
\hline Prepharynx & $\begin{array}{c}0.18-0.18 \times \text { X } 0.06- \\
0.06\end{array}$ & 0.018 & $0.046-0.050$ & \\
\hline Pharynx & $0.04-0.05 \times 0.1-0.1$ & $0.03 \times 0.09$ & $0.055-0.060$ & $0.05-0.12$ \\
\hline Esophagus & $\begin{array}{c}0.04-0.06 \times 0.08- \\
0.09\end{array}$ & & $0.161-0.182$ & $0.03-0.18$ \\
\hline Ovary & $\begin{array}{c}0.16-0.19 \times 0.22- \\
0.22\end{array}$ & $0.11 \times 0.09$ & $\begin{array}{c}0.230-0.260 \\
\times 0.184-0.165\end{array}$ & $\begin{array}{c}0.06-0.33 \text { X } 0.06- \\
0.34\end{array}$ \\
\hline Anterior Testis & $\begin{array}{c}0.28-0.35 \times 0.26- \\
0.31\end{array}$ & $0.21 \times 0.17$ & $\begin{array}{c}0.368-0.390 \\
X 0.253-0.260\end{array}$ & $\begin{array}{c}0.11-0.40 \times 0.13- \\
0.34\end{array}$ \\
\hline Posterior Testis & $\begin{array}{c}0.36-0.36 \text { X } 0.28- \\
0.30\end{array}$ & $0.23 \times 0.18$ & $\begin{array}{c}0.322-0.328 \mathrm{X} \\
0.391-0.396\end{array}$ & $\begin{array}{c}0.12-0.40 \times 0.11- \\
0.36\end{array}$ \\
\hline $\begin{array}{c}\text { Seminal } \\
\text { Receptacle }\end{array}$ & $\begin{array}{c}0.12-0.13 \times 0.32- \\
0.37\end{array}$ & & $\begin{array}{l}0.92-0.98 X \\
0.161-0.165 \\
\end{array}$ & \\
\hline $\begin{array}{l}\text { Uterus from } \\
\text { anterior } \\
\text { extremity }\end{array}$ & $1.26-1.27$ & & $1.84-1.95$ & \\
\hline $\begin{array}{l}\text { Uterus from } \\
\text { posterior } \\
\text { extremity }\end{array}$ & $1.08-1.11$ & & $0.92-0.98$ & \\
\hline
\end{tabular}

Thaparotrema pedicellatum [1-4] collected from intestine of and gallbladder of fishes Rita rita, Hemibagrus nemurus and Rita buchanani from Pakistan and Thailand varies from new species by fusiform body; widest at post-equatorial region of body; tegument spined; oral sucker rounded; pharynx rounded; testes oval to rounded in shape, anterior testis small and rounded, posterior testis large oval shape; ovary diamond shape; laurel canal present; uterine coils much compact, extending from posterior region of ventral to the ovary; vitelline follicles not close to lateral boundary of body.

Thaparotrema piscicola [1, 2, 4] collected from gallbladder of Gymnarchus niloticus from Africa varies from new species by tegument spined; oral sucker rounded; ventral sucker much broad; testes diagonal, branched; laurel canal present; vitelline follicles extending between pre-equatorial region and anterior testis; excretory bladder tube shape. 
Thaparotrema botswanensis [5, 6] collected from gallbladder of Clarias gariepinus from Africa varies from new species by widest at level of ventral sucker; tegument spined; oral sucker rounded to oval; ventral sucker smaller then oral sucker; pharynx rounded; caeca narrow, not overlapped by uterus; testes oval in shape; ovary bean shape at left side; seminal vesicle tubular commencing post-acetabular; seminal receptacle anterior to ovary; uterus intra-caecal; vitelline follicles not much compact, extra- caecal, fields between acetabular to ovary; excretory bladder with sigmoid between testes, pore terminal.

Table 2. Comparison of present species with closely related species of genus Thaparotrema discovered during current study

\begin{tabular}{|c|c|c|c|}
\hline Species & Present species & $\begin{array}{l}\text { Thaparotrema shamimi n. } \\
\text { sp. }\end{array}$ & $\begin{array}{c}\text { Thaparotrema } \\
\text { pedicellatum Verma } 1927\end{array}$ \\
\hline Host & Rita rita & Rita rita & $\begin{array}{l}\text { Rita rita andRita } \\
\text { buchanani }\end{array}$ \\
\hline Locality & Pakistan & Pakistan & Pakistan, Thailand \\
\hline Body & $\begin{array}{c}\text { Elongate, narrow, spindle } \\
\text { shape 5.6-5.36 }\end{array}$ & $\begin{array}{c}\text { Elongate, strongly curved at } \\
\text { level of ovary } 2.2-2.8\end{array}$ & $\begin{array}{c}\text { Fusiform shape, } 4.347- \\
4.530 \\
\end{array}$ \\
\hline Width & $\begin{array}{l}\text { Widest at post-equatorial } \\
\text { region } 0.5-0.6\end{array}$ & $\begin{array}{c}\text { Widest at equatorial region } \\
0.42-0.44 \\
\end{array}$ & $\begin{array}{l}\text { widest at post equatorial } \\
\text { region } 0.759-0.80\end{array}$ \\
\hline Oral Sucker & $\begin{array}{c}\text { Small, egg shape, terminal } \\
0.02-0.07 \times 0.16-0.17\end{array}$ & $\begin{array}{c}\text { Terminal, rounded 0.16-0.24 } \\
\text { X 0.15-0.17 }\end{array}$ & $\begin{array}{c}\text { Oval } 0.230-0.280 \times 0.138- \\
0.150\end{array}$ \\
\hline $\begin{array}{l}\text { Ventral } \\
\text { Sucker }\end{array}$ & $\begin{array}{c}\text { Wheel shaped and pre- } \\
\text { equatorial } 0.26-0.29 \times 0.22- \\
0.24\end{array}$ & $\begin{array}{c}\text { Oval, pre-equatorial } 0.17-0.18 \\
\text { X 0.16-0.17 }\end{array}$ & $0.115-0.135 \times 0.138-0.150$ \\
\hline Prepharynx & $\begin{array}{c}\text { Elongate } 0.18-0.18 \times 0.06- \\
0.06\end{array}$ & & $0.046-0.050$ \\
\hline Pharynx & Oval 0.04-0.05 X 0.1-0.1 & $\begin{array}{c}\text { Rounded } 0.07-0.08 \times 0.08- \\
0.10\end{array}$ & $\begin{array}{l}\text { Rounded to oval } 0.055- \\
0.060\end{array}$ \\
\hline Esophagus & $\begin{array}{c}\text { Esophagus short, broad } \\
0.04-0.06 \times 0.08-0.09\end{array}$ & $\begin{array}{c}\text { Esophagus elongate } 0.23-0.24 \\
\text { X } 0.09-0.10 \\
\end{array}$ & $0.161-0.182$ \\
\hline Ovary & $\begin{array}{l}\text { Triangular, anterolateral to } \\
\text { seminal receptacle } 0.16-0.19 \\
\text { X } 0.22-0.22 \\
\end{array}$ & $\begin{array}{c}\text { Heart shape, pre-semino- } \\
\text { receptacle } 0.16-0.17 \times 0.17- \\
0.20 \\
\end{array}$ & $\begin{array}{c}\text { Rounded, at right side of } \\
\text { body } 0.230-0.260 \times 0.184- \\
0.165 \\
\end{array}$ \\
\hline $\begin{array}{l}\text { Anterior } \\
\text { Testis }\end{array}$ & $\begin{array}{l}\text { Quarter-angular in form } \\
0.28-0.35 \times 0.26-0.31\end{array}$ & $\begin{array}{c}\text { Square shape } 0.24-0.29 \mathrm{X} \\
0.19-0.21 \\
\end{array}$ & $\begin{array}{c}\text { Rounded } 0.368-0.390 \\
\text { X0.253-0.260 }\end{array}$ \\
\hline $\begin{array}{l}\text { Posterior } \\
\text { Testis }\end{array}$ & $\begin{array}{c}\text { Penta-merous shape } 0.36- \\
0.36 \times 0.28-0.30\end{array}$ & $\begin{array}{c}\text { Triangle shape, posteriorly } \\
0.27-0.30 \times 0.21-0.31\end{array}$ & $\begin{array}{c}\text { Oval 0.322-0.328 X 0.391- } \\
0.396\end{array}$ \\
\hline $\begin{array}{c}\text { Seminal } \\
\text { Receptacle }\end{array}$ & $\begin{array}{c}\text { Pretesticular, tube shape, } \\
0.12-0.13 \times 0.32-0.37\end{array}$ & $\begin{array}{c}\text { Oval shape, pre-testicular } \\
0.07-0.09 \times 0.29-0.34\end{array}$ & $\begin{array}{c}\text { Rounded to oval 0.92-0.98 } \\
\text { X 0.161-0.165 }\end{array}$ \\
\hline $\begin{array}{l}\text { Seminal } \\
\text { Vesicle }\end{array}$ & & $\begin{array}{c}\text { Oval shape, at lateral side of } \\
\text { ventral sucker } 0.11-0.11 \mathrm{X} \\
0.05-0.07\end{array}$ & \\
\hline $\begin{array}{l}\text { Vitelline } \\
\text { follicle }\end{array}$ & $\begin{array}{l}\text { Laterally fields from } \\
\text { anterior region of ventral } \\
\text { sucker to ovary. }\end{array}$ & $\begin{array}{c}\text { Lateral fields from ventral } \\
\text { sucker to the seminal } \\
\text { receptacle }\end{array}$ & $\begin{array}{c}\text { Extending between } \\
\text { posterior of ventral sucker } \\
\text { and seminal receptacle }\end{array}$ \\
\hline
\end{tabular}


Thaparotrema shamimi [13] collected from gallbladder of Rita rita from Pakistan varies from new form by widest body of worm elongate; oral sucker rounded with curved at posterior; pharynx rounded in shape and attached to oral sucker; ventral sucker oval, large, pre-equatorial region of body; testes two diagonal, at posterior region of body, anterior testis square in shape and posterior testis triangle in shape, broad anteriorly with narrow posteriorly; ovary heart shape, vertical in position, pre-semino-receptacle left side of body; seminal receptacle oval in shape, vertical in position, pre-testicular; uterine coils highly condense, cover whole middle region of body, extended between posterior margin of ventral sucker to ovary; seminal vesicle oval in shape, at lateral side of ventral sucker.

\section{Conclusion}

The present trematodes belong to genus Thaparotrema on the basis of following differential characteristics including body shape and width, tegument without spines, suckers shape and position, prepharynx long and pharynx oval shape, testes shape and size, ovary heart shape, seminal receptacle shape and position, vitelline are in the form of small follicles and identified as new species Thaparotrema akbari. The name of new species denotes to the father's name of research scholar, Soofi Akbar.

\section{Authors' contributions}

Conceived and designed the experiments: $\mathrm{H}$ Soofi \& NA Birmani, Performed the experiments: H Soofi, Analyzed the data: $\mathrm{H}$ Soofi, NA Birmani, AM Dharejo \& AR Abbasi, Contributed materials/ analysis/ tools: H Soofi \& GS Ghachal, Wrote the paper: H Soofi \& NA Birmani.

\section{References}

1. Yamaguti S (1971.) Synopsis of digenetic trematodes of vertebrates. Keig Pub Co Toky Jap 1: 217-218.
2. Yamaguti S (1971). Synopsis of digenetic trematodes of vertebrates. Keig Pub Co Toky Jap 2: 507.

3. Soofi H, Birmani NA, Dharejo AM \& Abbasi AB (2015). First record of genus Thaparotrema Gupta, 1955 (Trematoda: Ophisthorchiidae) in Pakistan. $J$ Entomol Zool Stud 3: 232-234.

4. Bray RA, Gibson DI \& Jones A (2008). Keys to the trematode. CABI Pub Natur Hist Muse Lond 3: 9-35.

5. Rensburg van VCJ, As van JG \& King PH (2013). New records of digenean parasites of Clarias gariepinus (Pisces: Clariidae) from the Okavango Delta, Botswana, with description of Thaparotrema botswanensis sp. $\mathrm{n}$. (Plathelminthes: Trematoda). Afri Inver 54: 431-446.

6. Latifa GA, Khanum $\mathrm{H}$ \& Monwar $\mathrm{H}$ (2008). Helminth parasites of Polynemus paradiseus (Linnaeus, 1758). Bang J Zool 36: 35-42.

7. Bychowsky BE (1962). Monogentic Trematodes and Their Systametic and Phylogeny. Grap Art Press 242.

8. Read CP (1992.) Animal Parasitism. Inc Englw Clif New Jersey 265.

9. Ahmad AN \& Karimullah SSS (2014). Prevalence and morphology of helminth parasites of fish from River Swat, Khyber Pakhtunkhwa. Pak J Agric Res 27: 142-148.

10. Ayaz S, Khan MA, Rehman IU, Anwar M, Saeed S \& Zarin S (2013). Prevalence of Endoparasites in fresh water fishes in River Punjkorha, Khyber Pukhtunkhwa Pakistan. Int J Bio Pharm Allie Sci 2: 111-115.

11. Khanum H, Ferdows $\mathrm{J}$ \& Farhana R (2008). Community of helminth parasites in Rita rita (Hamilton Buchanun). J Biosci 16: 133-135.

12. Shakir HA \& Khan AM (2006). The prevalence of cestode infection in a freshwater catfish, Sperata Sarwari, 
Department of Zoology Punjab University, Lahore, Pakistan. Pun Uni J Zool 21: 41-47.

13. Soofi H, Birmani NA \& Dharejo AM (2016). Thaparotrema Shamimi new species in catfish Rita rita (Hamilton, 1822) from Jamshoro district Sindh, Pakistan. Inter J Adv Res Biol Sci 3(9): 124-129.

14. Soofi H, Birmani NA \& Dharejo AM (2016). Dendrorchis ritata n.sp.
(Trematoda: Gorgoderidae) from catfish Rita rita (Siluriformes: Bagridae) of Sindh, Pakistan. Inter J Faun Bio Stu 3: 17-19.

15. Garcia LA \& Ash LR (1979). Diagnostic Parasitology Clinical laboratory. West Ind Driv, St Louis Missouri, pp. 63141.

16. Schmidt GD (1988). Essentials of Parasitology. Brow Pub 2460 Keper Boul Dubuque 4(52001): 29. 